\title{
Elementos clave en el proceso de convivencia con la enfermedad de Parkinson de pacientes y familiares cuidadores
}

\author{
Key elements in the process of living with Parkinson's disease \\ for patiens and caregivers
}

\author{
A. Zaragoza Salcedo', J.M. Senosiain García', M. Riverol Fernández ${ }^{2}$, S. Anaut Bravo ${ }^{3}$, \\ S. Díaz de Cerio Ayesa ${ }^{4}$, M.E. Ursúa Sesma ${ }^{5}$, M.C. Portillo ${ }^{1}$
}

\section{RESUMEN}

Fundamento. La enfermedad de Parkinson produce un impacto considerable en la vida de las personas. Es necesario identificar los elementos clave que influyen en el proceso de convivencia con la enfermedad de Parkinson para que los profesionales de la salud puedan ayudar a los pacientes y sus familias a convivir lo mejor posible con los cambios y limitaciones producidos por la enfermedad.

Material y método. Se llevó a cabo un estudio cualitativo descriptivo. Este estudio se corresponde con la primera fase de un diseño exploratorio secuencial (Mixed-methods) que incluye a su vez una fase cuantitativa. Se realizó un proyecto multicéntrico. Para la recogida de datos se aplicó un muestreo de conveniencia y se utilizó una entrevista semi-estructurada realizada individualmente a pacientes y familiares cuidadores y dos cuestionarios para pacientes: la Escala de Hoehn \& Yahr y el Cuestionario PDNMS. Se realizó un análisis de contenido de las entrevistas y estadístico descriptivo de los cuestionarios.

Resultados. La muestra la constituyeron 46 participantes. Se identificaron tres elementos clave en el proceso de convivencia con la enfermedad de Parkinson: aceptación, adaptación y automanejo. Estos elementos condicionaron dos modos de convivencia con la enfermedad de Parkinson: una convivencia positiva, caracterizada por sentimientos de armonía, equilibrio, y naturalidad; y una convivencia negativa caracterizada por sentimientos de frustración, pérdida de control y autoestima.

Conclusiones. Es esencial que los profesionales de la salud conozcan a fondo estos elementos, así como los factores que los favorecen o dificultan. En la medida que se propicie la investigación en este ámbito y se identifiquen intervenciones efectivas se mejorará la atención integral de la personas en consonancia con las nuevas directrices para la cronicidad.

Palabras clave. Enfermedad crónica. Enfermedad de Parkinson. Convivencia. Metodología cualitativa.

\begin{abstract}
Background. Parkinson's disease has a considerable impact on people's lives. It is necessary to identify the key elements that influence the process of living with Parkinson's disease so that health professionals can help patients and their relatives to live as well as possible with the changes and limitations produced by the disease.
\end{abstract}

Material and methods. A qualitative descriptive study was realized. This study corresponded to the first phase of a sequential, exploratory design (mixed method) that in turn included a quantitative phase. A multicentre project was carried out. Convenience sampling was applied to collect data, a semi-structured interview was realized individually with patients and carer-relatives and two questionnaires with patients: the Hoehn $\&$ Yahr scale and the PDNMS questionnaire. Content analysis of the interviews and a statistical description of the questionnaires were used.

Results. The sample was made up of 46 participants. Three key elements were identified in the process of living with Parkinson's disease: acceptance, adaptation and self-management. These elements conditioned the modes of living with Parkinson's disease: positive living, characterized by feelings of harmony, balance and naturalness; negative living characterized be feelings of frustration, loss of control and self-esteem.

Conclusions. It is essential for health professionals to have a deep understanding of these elements, as well as of the factors that favor or hinder them. To the extent that research in this field progresses and effective interventions are identified, comprehensive patient care will be improved in consonance with the new directives for chronicity.

Key words. Chronic illness. Parkinson's disease. Living with. Qualitative methodology.
1. Universidad de Navarra. Departamento de Enfermería de la Persona Adulta. Pamplona

2. Clínica Universidad de Navarra. Departamento de Neurología. Pamplona

3. Universidad Pública de Navarra. Departamento de Trabajo Social. Pamplona

4. Asociación Navarra de Parkinson. Pamplona

5. Servicio Navarro de Salud-Osasunbidea. Centro de Salud de San Juan. Pamplona

\author{
Correspondencia: \\ $\mathrm{M}^{\mathrm{a}}$ Carmen Portillo \\ Universidad de Navarra \\ Departamento de Enfermería del Personal Adulta \\ Edif. Los Castaños \\ $\mathrm{C} /$ Irunlarrea, $\mathbf{s} / \mathbf{n}$ \\ 31008 Pamplona \\ E-mail.mportillo@unav.es
}

Recepción: 29 de septiembre de 2013

Aceptación provisional: 28 de noviembre de 2013

Aceptación definitiva: 13 de enero de 2014 


\section{INTRODUCCIÓN}

El aumento de la esperanza de vida, en gran medida consecuencia de los avances médicos y tecnológicos, lleva consigo también una mayor prevalencia de las enfermedades crónicas. Entre éstas, las enfermedades neurológicas se han convertido en una prioridad para la atención sanitaria en el mundo. En España hay más de siete millones de personas con alguna enfermedad neurológica grave $\mathrm{e}^{1,2}$.

Las enfermedades neurológicas que derivan en una discapacidad y/o dependencia, y en concreto la enfermedad de Parkinson (EP), producen un impacto considerable en la vida de las personas que las padecen, sus familias y la sociedad. Junto a los cambios físicos y psicológicos que experimentan los pacientes, se producen también cambios sociales en torno a los roles en el hogar y en el ámbito laboral. Estos procesos suelen afectar a las relaciones familiares íntimas, las relaciones sociales y en no pocas ocasiones llevan a un deterioro de la salud del cuidador ${ }^{3-12}$.

En conformidad con las directrices del Ministerio de Sanidad, Recursos sociales e Igualdad (2012) ${ }^{13}$, es necesario proporcionar a los pacientes con procesos crónicos y sus familiares una atención integral que aborde, junto a los aspectos físicos, las necesidades psicológicas, sociales, y familiares que derivan de esta situación. En este sentido, un campo de intervención para los profesionales sanitarios es el de ayudar a los pacientes a convivir lo mejor posible con las limitaciones y cambios producidos por la enfermedad.

Existe gran heterogeneidad en cómo los pacientes con enfermedades crónicas consiguen "acomodar" su vida a la enfermedad $^{14}$. Stanton y col ${ }^{14}$ han identificado algunos factores que propician esta adaptación como son el apoyo interpersonal que reciben los pacientes, algunos rasgos de la personalidad como el optimismo, el significado de la enfermedad para la persona y la repercusión de la misma en los valores y objetivos en la vida. Estos estudios se han realizado en pacientes con artritis reuma- toide, cáncer y enfermedades vasculares. Se necesita más investigación para confirmar los hallazgos en otras enfermedades crónicas como la EP y en los familiares cuidadores. Además, es necesario conocer a través de qué mecanismos estos factores influyen en el proceso de convivencia con la enfermedad.

Otros estudios ${ }^{15-18}$ han explorado la adaptación y convivencia con la diabetes y la esclerosis múltiple. Han identificado que la convivencia con una enfermedad crónica se caracteriza por ser un proceso de adaptación desde una etapa denominada extraordinaria (extraordinariness) a una etapa de normalización (ordinariness). La etapa extraordinaria se caracteriza por la sensación de pérdida de control y de la identidad personal, y por la agudización de los cambios producidos por la enfermedad. Esta etapa extraordinaria suele coincidir con los comienzos de la enfermedad o cuando se produce una exacerbación de los síntomas. La etapa de normalización se alcanza cuando los pacientes logran integrar la enfermedad en su vida y en su rutina diaria y se caracteriza porque la persona es capaz de manejar y llevar un cierto control sobre sus respuestas a la enfermedad, desarrollando sentimientos de armonía, equilibrio, orden, capacitación y autocuidado. Este proceso de convivencia y adaptación a la enfermedad es complejo, dinámico y cíclico. En muchos casos los pacientes pueden conseguir un rol activo en el manejo de la enfermedad ${ }^{18}$. Aunque estos estudios mencionados han identificado estas dos etapas extremas, queda todavía por explorar y conocer cómo se produce esa transición desde la etapa extraordinaria a la etapa de normalización, qué factores están afectando ese cambio y de qué modo actúan.

El objetivo principal de este trabajo es explorar en profundidad el proceso de convivencia con la EP de pacientes y familiares cuidadores. Concretamente, se busca identificar y conocer los elementos que integran este proceso de convivencia y la repercusión de los mismos en el modo de convivir con la EP. 
Este estudio forma parte de un programa de investigación denominado ReNACE cuyas siglas significan: Recuperación, Normalización; Aceptación y Convivencia con la enfermedad. A través de este programa de investigación se pretende fomentar la integración de la enfermedad crónica en la vida de pacientes y familiares a través del diseño, implantación y evaluación de intervenciones multidisciplinares individualizadas.

\section{MATERIAL Y MÉTODOS}

Para responder al objetivo del estudio, se ha realizado un estudio cualitativo descriptivo. Este estudio cualitativo constituye la primera fase de un proyecto con diseño exploratorio secuencial (Mixedmethods) $)^{19-21}$.

Se planteó un proyecto multicéntrico y la recogida de datos se llevó a cabo en la Zona Básica de Salud de San Juan (Pamplona) del Servicio Navarro de Salud-Osasunbidea; la Consulta de Neurología y la Unidad de Trastornos de Movimiento de la Clínica Universidad de Navarra; y la Asociación Navarra de Parkinson (ANAPAR) con investigadores de todos los centros implicados en el proyecto.

Tras obtener la aprobación del Comité de Ética de la Universidad de Navarra y la autorización de cada centro implicado en el estudio, se reclutaron pacientes con EP y sus familiares cuidadores a través de un muestreo de conveniencia ${ }^{22}$. Los criterios de inclusión fueron: pacientes en cualquier estadio de enfermedad y un familiar cuidador que conviviera con el paciente o que mantuviera una relación estrecha y colaborase activamente en el cuidado; que desearan participar voluntariamente en el estudio; mayores de 18 años; de lengua castellana o inglesa; y residentes en Navarra.

Con el objetivo de proteger en todo momento la confidencialidad de los participantes se utilizaron diferentes medidas. La información acerca del estudio y el acceso se llevó a cabo desde los propios centros participantes, bien a través de cartas in- formativas (centro de Salud de San Juan y ANAPAR) o directamente a través del médico responsable (CUN). Los pacientes y familiares cuidadores interesados en participar enviaron el consentimiento informado y su teléfono al equipo investigador para ser contactados.

Otra estrategia para asegurar la confidencialidad de los datos fue la de utilizar códigos para nombrar a los participantes. Estos códigos incluyeron tres elementos:

- P o F: paciente o familiar;

- ANAPAR, CSJ o CUN; según el centro de procedencia.

- Número: referencia al orden de inclusión en el estudio en cada uno de los centros participantes.

Para la primera fase del estudio la recogida de datos se completó en noviembre de 2012. Esta recogida se llevó a cabo principalmente a través de una entrevista semiestructurada grabada que se realizó de forma individual con pacientes y familiares cuidadores, la mayoría de las veces en sus domicilios. Los temas tratados en las entrevistas se presentan en la tabla 1. Además para los pacientes se emplearon dos cuestionarios: la escala de Hoehn \& Yahr ${ }^{23,24}$; y el Cuestionario de Síntomas no Motores en EP (PD-NMS Quest) ${ }^{25,26}$.

Siempre que fue posible, las entrevistas se realizaron sin que estuviera presente el otro participante, es decir el familiar cuidador en caso del paciente o el paciente en caso del familiar cuidador. Al comienzo de cada entrevista se les recordó el objetivo de la misma, conocer su experiencia con la EP, bien como paciente, bien como familiar-cuidador. La participación y respuesta de los participantes durante las entrevistas fue muy positiva. En la mayoría de los casos manifestaron su agradecimiento por la oportunidad de compartir su situación y experiencia. En alguna ocasión algún participante se emocionó visiblemente en la entrevista. En todos estos casos por respeto a la intimidad de la persona y por delicadeza con ella, se pararon las grabaciones. Fueron los propios participantes los que, una vez repuestos, manifestaron su deseo de proseguir con la recogida de datos. 
Tabla 1. Entrevista semiestructurada con pacientes y familiares/cuidadores

Tema 1. Evolución del proceso de la EP
¿Cuándo le diagnosticaron la EP? ¿Qué ocurrió cuando le diagnosticaron de EP? o ¿Qué ocurrió
cuando le diagnosticaron a su familiar de EP? ¿Cómo ha sido este tiempo hasta ahora?

\section{Tema 2. Cambios ocasionados por la enfermedad}

¿Cómo le ha influido o está influyendo esta enfermedad en su vida? ¿Piensa que su vida le ha cambiado o va a cambiar en algún sentido por la EP? ¿Qué importancia tiene esto para usted?

Tema 3. Alternativas a los cambios e implicación en el proceso. Factores que influyen o han influido en la convivencia con la enfermedad

En relación a los cambios producidos por la EP, ¿Qué ha hecho para sentirse mejor, o para responder o llevar mejor estos cambios? ¿Qué le ha ayudado? ¿Qué cosas le han dificultado o no le han ayudado? ¿Qué es lo que más le cuesta de su enfermedad?

\section{Tema 4. Situación actual de aceptación, adaptación e integración de la enfermedad en sus vidas \\ ¿Cómo se siente en relación a la EP? ¿Cómo lleva la EP en su vida? ¿Considera que está manejando la situación? ¿Qué sentido/significado tiene para usted?}

Tema 5. Fuentes y redes de apoyo/sociales disponibles

¿Cuenta con algún tipo de apoyo en su situación? ¿Cómo describiría ese apoyo?

\section{Tema 6. Sentido de coherencia en su vida y satisfacción con la vida}

En su vida, ¿Cómo maneja/ha manejado una situación complicada o contratiempos? ¿Qué cosas son importantes en su vida? ¿Cómo se siente con su vida?

\section{Final de la entrevista}

¿Le gustaría añadir algo más?

Tras la realización de cada entrevista se procedió a la transcripción íntegra de las mismas. El análisis se llevó a cabo siguiendo el modelo de Miles y Huberman ${ }^{27}$ de análisis de contenido. El análisis consistió en una codificación del texto. Para ello no se siguió un proceso descriptivo de identificación y cuantificación de categorías, sino un proceso interpretativo. De acuerdo a este proceso cada entrevista se analizó partiendo del marco reconocido en la literatura sobre la convivencia con la enfermedad ${ }^{14-18}$ el cual dirigió la identificación de categorías. Se buscó reconocer cómo estaba siendo el proceso de convivencia con la enfermedad desde la experiencia y significado de cada participante (paciente o familiar cuidador) y a partir de ahí reconocer patrones comunes.

Tres investigadores analizaron de forma independiente y manualmente los tres primeros pares de entrevistas, pacientefamiliar cuidador. Tras el análisis de cada pareja de entrevistas, se realizaron sesiones de debate y consenso de las categorías emergentes y se procedió a definir cada una de ellas con el fin de facilitar su identificación en las restantes entrevistas. Posteriormente, cada uno de los tres investigadores procedió al análisis de diferentes entrevistas. Se mantuvieron regularmente sesiones para la depuración y síntesis de las categorías.

Al final de este proceso se vio necesario incluir en el estudio algún participante más con un perfil positivo en cuanto a la convivencia con la enfermedad con el fin de completar explorar de aspectos relacionados con una buena convivencia. A través del médico responsable de CUN se puedo acceder a dos parejas de participantes con estas características. Este tipo de estrategia, es decir, los muestreos de conveniencia, son muy adecuados en la investigación cualitativa, ya que la muestra debe facilitar el estudio de los fenómenos de interés y 
ayudar así a la generación de hipótesis ${ }^{22}$. Por otro lado, el tamaño de la muestra no se determinó a priori sino que se decidió de acuerdo a la "saturación teórica de los datos" ${ }^{28}$. De este modo, se fueron incluyendo participantes en el estudio hasta asegurarse que ya no se identificaban nuevas categorías.

Durante el proceso de análisis se utilizó el programa informático Nvivo ${ }^{\circledR}$ de NUD*IST (QSR) 9.2 que permite el manejo de la información, la organización de las categorías, y su integración para la identificación de temas emergentes. Además, a lo largo de este proceso, se fueron verificando (host verification) los resultados del análisis con participantes (pacientes y familiares cuidadores) y profesionales implicados en su atención a través de charlas informales divulgativas del proyecto. Por otro lado se llevaron a cabo cuatro sesiones de presentación y validación de resultados con los miembros del equipo multidisciplinar del programa ReNACE formado por enfermeras del ámbito académico con conocimiento y experiencia en el cuidado de pacientes neurológicos, médicos, uno de ellos neurólogo y dos trabajadoras sociales.
A su vez, se llevó a cabo un análisis descriptivo de los datos obtenidos por medio de los cuestionarios, utilizando el programa SPSS 15.0.

\section{RESULTADOS}

\section{Características de la muestra}

Un total de 46 participantes formaron la muestra del estudio: 22 parejas de paciente y familiar cuidador; un paciente sin familiar cuidador por carecer de él; y un familiar cuidador sin paciente por fallecimiento del mismo durante la recogida de datos. De los 46 participantes, tres pacientes no pudieron participar en la entrevista grabada por deterioro cognitivo.

Como puede verse en la tabla 2, los pacientes de la muestra tenían una afectación motora leve y una afectación no motora moderada. Un $65 \%$ de la muestra de pacientes lo constituyeron varones con una mediana de edad de 73 años. Un $74 \%$ de la muestra de familiares /cuidadores fueron mujeres, en la mayoría esposas de los pacientes, con una mediana de edad de 65 años.

Tabla 2. Características sociodemográficas de la muestra

\begin{tabular}{lll}
\hline \multicolumn{1}{c}{ Variables } & \multicolumn{1}{c}{ Pacientes* $^{*}$} & \multicolumn{1}{c}{ Familiares/cuidadores** $^{*}$} \\
\hline Edad & $73(65,76,25)$ & $65(59,50,74)$ \\
\hline Sexo & Varón: $15(65,2 \%)$ & Varón: $6(26,1 \%)$ \\
& Mujer: $8(34,8 \%)$ & Mujer: $17(73,9 \%)$ \\
\hline \multirow{2}{*}{ Estado civil } & Soltero: $2(8,7 \%)$ & Soltero: $2(8,7 \%)$ \\
& Casado/pareja: $20(87 \%)$ & Casado/pareja: $21(91,3 \%)$ \\
& Viudo: $1(4,3 \%)$ & \\
\hline \multirow{2}{*}{ Area de residencia } & Rural: $4(17,4 \%)$ & Rural: $4(17,4 \%)$ \\
& Semirural: $2(8.7 \%)$ & Semirural: $1(4,3 \%)$ \\
& Urbana: $17(73.9 \%)$ & Urbana: $18(78,3 \%)$ \\
\hline Hoehn y yahr & 2,$5 ;(1,5 ; 4)$ & \\
Pd-nmsquest & $12 ;(6,75 ; 15)$ & \\
\hline
\end{tabular}

Valores Edad, Hoehn y Yahr y PD-NMSQuest: Mediana y Rango Intercuartil (IQR): Percentiles 25/75 (P25, P75).

* Pacientes $\mathrm{n}=23$,

** Familiares/Cuidadores $\mathrm{n}=23$ 


\section{Categorías identificadas}

Como resultado del análisis se identificaron 4 categorías principales y 11 subcategorías que se presentan en la tabla 3.

Para responder al objetivo planteado en este trabajo de identificar cómo es el proceso de convivencia con la EP de pa- cientes y familiares cuidadores, se presentan los resultados correspondientes a las categorías siguientes:

- Categoría 1. Elementos que intervienen en la convivencia con la EP

- Categoría 2. Modos de convivencia con la EP

Tabla 3. Categorías y subcategorías identificadas tras el proceso de análisis

\begin{tabular}{ll}
\hline \multicolumn{1}{c}{ Categorías } & \multicolumn{1}{c}{ Subcategorías } \\
\hline $\begin{array}{l}\text { Categoría 1. Elementos que intervienen en la } \\
\text { convivencia con la EP }\end{array}$ & $\begin{array}{l}\text { Aceptación } \\
\text { Adaptación } \\
\text { Automanejo }\end{array}$ \\
\hline Categoría 2. Modos de convivencia con la EP & $\begin{array}{l}\text { Convivencia positiva } \\
\text { Convivencia negativa }\end{array}$ \\
\hline & $\begin{array}{l}\text { Respuestas de afrontamiento } \\
\text { Recursos } \\
\text { Tratamiento } \\
\text { Síntomas motores y no motores }\end{array}$ \\
\hline de convivencia con la EP & $\begin{array}{l}\text { Respuestas y experiencias de la persona con la EP } \\
\text { Salud del Familiar/cuidador }\end{array}$ \\
\hline $\begin{array}{l}\text { Categoría 4. Repercusiones de la enfermedad en proceso } \\
\text { la vida del paciente y familiar/cuidador }\end{array}$ &
\end{tabular}

A continuación se presenta en detalle cada una de las categorías. Se han introducido fragmentos de entrevista para ilustrar los resultados enunciados.

\section{Categoría 1: Elementos que intervienen en la convivencia con la EP}

Durante las entrevistas y su posterior análisis se pudieron identificar tres elementos clave en el proceso de convivencia con la EP: aceptación, adaptación y automanejo. Estos elementos se encontraron tanto en los pacientes como en los familiares cuidadores, si bien no siempre de forma simultánea. Seguidamente se describe cada uno de los elementos.

\section{Aceptación}

Como pudo apreciarse en la mayoría de las entrevistas, la aceptación o no de la enfermedad fue mencionada por los par- ticipantes como un aspecto clave en su proceso de convivencia con la EP. Se describe como un proceso interno y libre de la persona a través del cual ésta reconoce su realidad y la asume. Los participantes la consideraban condición necesaria y el primer paso para llevar bien la EP y sus consecuencias. El siguiente testimonio de uno de los familiares cuidadores muestra este punto:

FCSJ5: "para mi la palabra clave es aceptar, si no se acepta mal vamos. Porque eso efectivamente repercute en la familia y repercute en todo, en tu salud, en tu estado de ánimo, en tu trabajo y en todo lo que te rodea, eso está claro, pienso yo"

De las entrevistas se identificaron tres tipos de aceptación que se han denominado en este estudio: pseudo-aceptación; resignación y aceptación positiva. La primera consiste en una aceptación aparente de la enfermedad sin asumir realmente sus consecuencias. Se identificó en algunos pacientes en estadios iniciales de la EP o con 
sintomatología todavía leve. La aceptación resignada es aquella en la que la persona reconoce su realidad y entiende que es mejor no revelarse ante ella. Este tipo de aceptación apareció con mucha frecuencia en las entrevistas, tanto en pacientes como en sus familiares cuidadores. Por último, la aceptación positiva es aquella en la que la persona manifiesta un deseo activo de identificarse con la nueva situación y de implicarse poniendo los medios necesarios. Esta última, se identificó en varios participantes con características comunes como fueron: haber acomodado sus expectativas de vida a su nueva situación, vivir con esperanza su enfermedad y limitaciones, y haber descubierto aspectos positivos en esta situación. El siguiente fragmento de entrevista muestra este punto:

PANAPAR13: "empecé por llamarle a la enfermedad que era una 'amiga'... porque no todo lo que me ha traído es malo, sino que también me ha traído aspectos positivos, ¿no? ...que empiezas a valorar más a la familia, los amigos, la naturaleza... tienes más aficiones... y si eres cristiano, como tengo la suerte de ser yo, porque tengo el regalo de la fe, pues también es una ocasión de acercarte más a Dios... Una desgracia puede ser una oportunidad, según como te la tomes, puede cambiarte la vida, te cambia y en muchos aspectos a mejor...".

Además de los tres tipos de aceptación, se identificó la situación de "no aceptación", es decir, aquella en la que la persona se revela contra su situación centrándose en los problemas y pérdidas y no en la búsqueda de soluciones y/o alternativas. En estas situaciones, que no fueron muchas, los pacientes reflejaron encontrarse en un estado de desesperanza y cierto aislamiento, tal y como se refleja en el siguiente ejemplo:

PANAPAR6: "El mero hecho de no poder hacer cosas que antes eran normales en mi vida. Eso me cuesta mucho aceptar. La aceptación es lo que me cuesta a mi... pues por ejemplo, el no poder viajar como yo quisiera, como antes... Hay que ir a tal sitio, pero hay que ir con el coche, pues no puedo, y de hecho te dificulta mucho el movimiento. Me siento menos libre que antes, mucho menos libre. Me he metido como en mi zulo, como en mi zulo, ¿no?, en mi agujero, y ahí estoy metido".

\section{Adaptación}

La mayoría de los participantes reflejaron que con el trascurso del tiempo fueron aprendiendo a vivir en su nueva situación, a llevar la enfermedad, a asimilarla. Este elemento se denominó en el presente estudio: adaptación. Los participantes afirmaron que el hecho de que la enfermedad se presentara de forma gradual ayudó en ese proceso de asimilación y adaptación.

Principalmente de las entrevistas de familiares se pudieron identificar elementos facilitadores de la adaptación, tales como el conocimiento acerca de la enfermedad, las respuestas del paciente, el tratamiento y sus efectos. También se identificó en dos casos cómo la adaptación de las expectativas y objetivos de vida a la realidad favorecían este proceso.

FANAPAR11: "pues ahora me encuentro como más tranquila, como más adecuada a lo que me toca. Al principio me rebelaba más con lo que me pasaba, pero ahora es que me parece a mí que..., que me he ido adaptando a esta situación y es que lo que quiero es, como él, tranquilidad y paz, ante todo. Y disfrutar... cuando vienen los hijos y de esas pequeñas cosas".

Algunos participantes, tanto pacientes como sus familiares cuidadores, manifestaron cómo la agudización de los síntomas dificultaba la adaptación y que, sin embargo, ésta era más fácil cuando no había muchos síntomas limitantes. También se percibió en varias entrevistas cómo la ayuda de los familiares cuidadores fue clave en esa adaptación, no dejando a su ser querido aislarse o pararse:

FCSJ1: "yo lo único que le animo es a eso: No te tienes que dejar; tú lo que tienes que hacer es por la mañana, si puedes, andar media hora, pues media hora; si puedes andar una hora, pues una hora; hacer un poquito de gimnasia, hacer unos ejercicios, haces un poco de bicicleta. Todo para que, bueno, si luego mañana va a más pues no tienes más remedio, pero hoy por hoy si puedes retrasarlo, pues intentas retrasar". 


\section{Automanejo}

A través de las entrevistas se identificó cómo algunos pacientes y familiares cuidadores durante el transcurso de la enfermedad, aprendieron a gestionar y manejar su situación con iniciativa y cierta independencia, en definitiva, a llevar las riendas con motor propio. A esta cualidad se le denominó automanejo.

Varios pacientes manifestaron esta capacidad de automanejo en el hecho de hacer frente a los problemas, buscando soluciones con iniciativa. En general, coincidían en ser personas con energía, luchadoras, satisfechos con su vida. Percibían que controlaban la enfermedad y no al revés. Se sentían interiormente libres aunque físicamente no siempre pudieran manejarse con autonomía. El ejemplo siguiente muestra esta actitud proactiva:

PCSJ2: "Si, pero no a peor, yo creo que a mejor. Como que me he hecho más... no fuerte, pero... no sé, pues que ves las cosas de otra manera. Pienso cosas distintas. Pienso, por ejemplo, si uno tiene un problema pues piensa: 'vaya, tengo esto y no sé qué...', y yo pienso en cómo se puede arreglar, por ejemplo, en vez de, o sea como que buscas soluciones en vez de estar pensando en el problema que tienes".

Aunque esta capacidad de iniciativa y proactividad global fue especialmente evidente en varios casos, bastantes participantes manifestaron también un aprendizaje y automanejo en ámbitos concretos como el uso de la medicación, el ejercicio físico y la movilidad. En algunos casos fue el propio paciente quien manifestó este control y en otros fue el familiar cuidador. En todos ellos se apreció cómo esta capacidad ayudaba a llevar mejor la situación.

\section{Categoría 2: Modos de convivencia con la EP}

Tras el análisis de las entrevistas se lograron identificar en detalle dos modos extremos de convivencia con la EP: convivencia positiva y convivencia negativa. También se constató a través de las entrevistas que la mayoría de los participantes se encontraban en alguna situación entre ambos extremos. Dada la relevancia de estos dos modos de convivencia, se detalla a continuación cada uno de ellos.

\section{Convivencia positiva}

Varios participantes del estudio, pacientes y familiares cuidadores, mostraron a través de las entrevistas una serie de características en el modo de convivir y llevar la enfermedad, caracterizada por sentimientos de armonía y equilibrio en sus vidas, una actitud positiva ante los cambios requeridos por la nueva situación y naturalidad en el modo de llevar las limitaciones. Estas características son las que en este estudio definen una convivencia positiva. Se ha constatado que los participantes que mostraron estos signos fueron los mismos que reflejaron una aceptación positiva, una buena adaptación y un buen automanejo. El siguiente fragmento muestra estas ideas:

FCSJ8: "Me compré una bicicleta cuando estaba bien [se refiere a su esposa, la paciente] y me recorrí toda la zona limítrofe de Pamplona, pero eso ya se ha acabado también, se ha terminado, pero hay que aceptarlo y ya está. Así de sencillo ...y después pues la vida normal, el aceptar lo que tengo y ser feliz con lo que tengo en casa, y con eso me basta... Yo no echo en falta nada, ni estoy desesperado porque la veo así y no puedo irme o no puedo hacer... no, no, no, para mí eso no es un problema".

También se evidenció que muchos pacientes con EP durante etapas iniciales, en las que todavía no tenían sintomatología importante, mostraron signos de una convivencia positiva. En algunos de esos casos, al desarrollarse más la enfermedad y aparecer nuevas limitaciones, se puso de manifiesto que no se había dado realmente una aceptación, sino más bien pseudo aceptación.

\section{Convivencia negativa}

De forma contraria a lo expuesto en el apartado anterior, varios participantes manifestaron vivir, como consecuencia de la enfermedad y sus limitaciones, una 
situación caracterizada por sentimientos de frustración, pérdida de control y autoestima. Estas características son las que en este estudio definen una convivencia negativa con la EP.

Se identificó esta situación en casos de participantes con sintomatología importante, limitante, y que la persona no conseguía controlar. También se apreció en familiares cuidadores que percibían que las demandas de cuidado estaban siendo mayores que las posibilidades de cuidado. En estos casos, el familiar cuidador, habitualmente mujer, se encontraba abrumada, cansada y sin poder responder adecuadamente a las demandas del familiar atendido y de su propia vida.

Asimismo este modo de convivencia se presentó en algún participante con afectación leve y pocos años de evolución de la enfermedad, como es el caso que muestra el siguiente fragmento de entrevista:

PANAPAR12: "Y aún ahora, y cada vez que hablo con cualquiera me deprimo total, lo estoy pasando fatal... Pues por ejemplo yo subirme a quitar una cortina no puedo. Subirme a limpiar las baldosas tampoco puedo, No me quiero hacer a la idea de que eso tiene que vivir toda la vida conmigo... Y hay cosas que ya no me hacen ilusión ni de vivir ni al verme tan limitada pues no me siento satisfecha. Siento que me falta algo. Algo que tenía y no lo tengo".

Finalmente, los participantes con una convivencia negativa son los mismos que reflejaron una gran dificultad para aceptar la situación que estaban viviendo, así como para adaptarse y mantener un adecuado automanejo.

PANAPAR8: "llegas a un momento que estás todo el día dándole vueltas a las cosas y sin querer pues piensas que eres un inútil y eres un estorbo, un estorbo para todos... yo para mí creo que no se adapta nadie. De la boca para afuera sí pero... pero la realidad, la realidad es, es, es eso ¿no?"

\section{DISCUSIÓN}

Este estudio ha identificado tres elementos clave en el proceso de convivencia con la EP: aceptación, adaptación y auto- manejo. Entre los elementos identificados, la aceptación es el primero y más relevante, ya que va a condicionar todo el proceso posterior. La aceptación es una cuestión interna y libre de la persona, a través de la cual ésta reconoce su nueva realidad, la asume y se enfrenta a ella. Hay que tener en cuenta que se han encontrado distintos niveles de aceptación y que la aceptación efectiva es aquella que asume la realidad que se vive, buscando identificarse con ella y haciendo frente a sus consecuencias.

El proceso de aceptación está influido principalmente por "el modo de mirar" la $\mathrm{EP}$, es decir, aquellos pacientes o familiares cuidadores que se focalizan en la enfermedad y en lo que ya no pueden hacer tienen más dificultades en la aceptación que aquellos otros que saben mirar de forma positiva y esperanzada. Estos resultados confirman los hallazgos de Stanton y col ${ }^{14}$ acerca de la influencia de las personalidades optimistas en la adaptación psicológica a la enfermedad crónica.

La adaptación es otro de los elementos que forman parte del proceso de convivencia con la EP. Este segundo elemento es una continuación del proceso anterior, a través del cual la persona va aprendiendo a vivir con y en su nueva situación. Está favorecido por un control de los síntomas de la enfermedad, el conocimiento acerca de la EP y las respuestas de la persona a la misma, la adecuación de las expectativas de vida y el apoyo familiar.

Estos factores favorecedores de la adaptación se han encontrado también en otros estudios. En concreto Whittemore ${ }^{29}$, ha identificado la relevancia de la estabilización o control de síntomas como factor para la adaptación. Stanton y col ${ }^{14}$ identificaron como factores determinantes en la adaptación, además de la personalidad, ya mencionada, las relaciones interpersonales, la percepción acerca de las implicaciones de la enfermedad para los valores y expectativas de vida y las respuestas de afrontamiento.

El tercer elemento presente en el proceso de convivencia es el automanejo, es decir, el manejo de la enfermedad, del tratamiento y de las respuestas, con iniciativa 
y cierta independencia, en otras palabras, llevar las riendas de la situación. La relevancia e influencia de este elemento para la convivencia con la enfermedad ya se está señalando en la literatura ${ }^{30}$. En el presente estudio se ha destacado que personalidades proactivas y luchadoras han conseguido un mayor automanejo de la EP. Estos resultados confirman los hallazgos de Stanton y $\mathrm{col}^{14}$ que indican que llevan mejor la enfermedad crónica personas con respuestas de afrontamiento activas, es decir, que buscan: información, soluciones a los problemas y apoyo social.

Además de estos tres elementos mencionados, el presente estudio también ha puesto de manifiesto la relación que existe entre estos elementos y el modo de convivencia con la EP, es decir, dependiendo de cómo la persona acepte, se adapte y maneje su enfermedad y sus consecuencias, logrará convivir mejor o peor con la EP.

Las características de la convivencia positiva o negativa identificadas en este estudio son las mismas que las características de las etapas de normalización y extraordinaria identificadas en la literatu$\mathrm{ra}^{15-18}$. Así, estos autores, describen la etapa extraordinaria como aquella caracterizada principalmente por una pérdida de la identidad personal y autoestima, sentimientos de inseguridad y vulnerabilidad, y pérdida de control de la situación. En la etapa ordinaria, sin embargo, prevalecen los sentimientos de armonía, control, equilibrio y reconstrucción de la propia identidad. En contraste con estos autores, el presente estudio ha puesto de manifiesto que esas situaciones, más que etapas del proceso de convivencia, deben considerarse como resultados de la misma. Así, no todas las personas pasan necesariamente por una convivencia negativa antes de conseguir una buena convivencia con la enfermedad, ni tampoco todas las personas llegan a convivir positivamente con ella. Otro resultado destacable de este estudio, en consonancia con los hallazgos de los citados autores, es que el proceso de convivencia, tal y como se ha señalado anteriormente, requiere la capacidad consciente de la persona de trascender la presente situación, es decir, de mirar más allá de los límites y confinamientos de la realidad presente. Este proceso está mediado por cualidades psicosociales y espirituales de la persona entre las que se encuentra la esperanza, el optimismo y el espíritu de lucha.

\section{Limitaciones del estudio}

Los resultados de este estudio provienen de la fase cualitativa de un proyecto de investigación combinada, tal y como se ha indicado en el apartado de metodología de este artículo. La investigación cualitativa tiene como finalidad explorar y conocer en profundidad el fenómeno de estudio y, por tanto, no persigue la generalización de los resultados como se pretende en los estudios experimentales. En el caso presente se buscaba comprender el fenómeno de la convivencia con la EP y se ha identificado que éste está mediado por los procesos de aceptación, adaptación y automanejo. Es necesario seguir indagando en los factores que propician estos procesos ya que darán la clave para diseñar intervenciones eficaces que fomenten la convivencia positiva con la enfermedad. Precisamente este es el objetivo de la segunda parte del proyecto que aquí se ha presentado. Por otro lado, es urgente trabajar también en el diseño de instrumentos que midan en la práctica el grado de convivencia con la EP de pacientes y familiares cuidadores. Este instrumento será esencial para poder evaluar intervenciones en este ámbito.

El proyecto se llevó a cabo en la comunidad foral de Navarra. Es muy posible que las características socio-demográficas y culturales de esta región hayan influido en el estudio. La mayoría de los participantes provienen de familias estructuradas, de clase media y que entienden el cuidado como un valor. Se precisan estudios en otros contextos socio-económicos y culturales que ayuden a confirmar o identificar nuevos factores.

Por otro lado, la muestra de los pacientes participantes contaba con una afectación motora leve y no motora moderada. Es necesario replicar este estudio con muestras de pacientes con una media de afecta- 
ción motora más severa con el fin de confirmar la validez de los presentes resultados. Sin embargo, hay que tener en cuenta las dificultades metodológicas que puede entrañar el indagar acerca de la experiencia de vivir con la EP en pacientes en estadio avanzado, teniendo en cuanta el deterioro físico (logopédico, motor...) y cognitivo que pueden tener estos pacientes.

El presente estudio se ha llevado a cabo por un equipo multidisciplinar de investigadores y profesionales que han intervenido en las distintas etapas del estudio. Este hecho ha requerido y propiciado un proceso de reflexión y discusión a lo largo de todo el proceso de la investigación que sin duda contribuye a la validez de los resultados. Cabe también añadir como factor positivo, el conocimiento y experiencia del equipo tanto en investigación cualitativa como en el área de los procesos neurológicos entre los que se incluye el Parkinson.

\section{Implicaciones del estudio para la práctica clínica}

La atención integral a las personas que padecen enfermedades crónicas y sus familiares requiere un abordaje que contemple a la persona en su totalidad y no sólo la enfermedad y sus síntomas ${ }^{13}$. Este tipo de acercamiento supone un cambio de paradigma con respecto al modelo biomédico, centrado mayoritariamente en la enfermedad, sus causas y tratamiento. Como se ha identificado en este estudio y se comprueba en la experiencia cotidiana trabajando al lado de enfermos y familiares, cada persona vive y responde a la enfermedad de forma única y personal. Algunas personas consiguen una adaptación y convivencia positiva mientras que otros no lo logran. En el primer caso, las personas se encuentran en armonía, paz y control de su situación, mientras que en el segundo caso predominan los sentimientos de frustración, pérdida de control y autoestima. El llegar a un resultado u otro, como se ha visto, no depende únicamente de un buen control de síntomas sino que intervienen otros elementos como son la aceptación de la enfer- medad y sus consecuencias, la adaptación a la misma y el automanejo.

Es esencial, por tanto, que los profesionales de la salud conozcan a fondo estos elementos, así como los factores que los propician o dificultan, que sepan valorar e identificar su presencia o ausencia en los pacientes y familiares y sepan intervenir de forma eficaz. En la medida que se propicie la investigación en este ámbito y se identifiquen intervenciones efectivas se mejorará la atención integral de la personas, en consonancia con las nuevas directrices para la cronicidad ${ }^{13} \mathrm{y}$ con ello, se contribuirá a una mejor adecuación y eficiencia de los recursos socio sanitarios.

\section{Agradecimientos}

Los autores del presente estudio agradecen en primer lugar a los pacientes con EP y sus familias que han participado en el presente estudio. Además se agradece la colaboración de las instituciones participantes: Asociación Navarra de Parkinson (ANAPAR); Departamento de Neurología de la Clínica Universidad de Navarra; Centro de Salud de San Juan de Osasunbidea y por último, a la Universidad de Navarra y la Sociedad Española de Enfermería Neurológica (SEDENE) que han hecho posible el presente estudio.

\section{BIBLIOGRAFÍA}

1. Benito-León J, Bermejo-Pareja F, Morales-González J, Porta-Etessam J, Trincado R, Vega S et al. Incidence of Parkinson disease and Parkinsonism in three elderly populations of central Spain. Neurology 2004; 62: 734-741.

2. Gómez Ibáñez A, Irimia P, Martinez-Vila E. The problem of neurological emergencies and the need for specific neurology shifts. An Sis Sanit Navar 2008; 31: 7-13.

3. Portillo Vega MC, Wilson-Barnett J, Saracibar RÁzQUin MI. Análisis de un marco conceptual para el proceso de participación informal en el cuidado después de un ictus. Enferm Clín 2002; 12: 143-151.

4. Thomas S, Sweetnam C. Parkinson's disease. Caring for the carers. Prim Health Care 2002; 12: 27-31. 
5. Portillo MC, Corchón S, López-Dicastillo O, Cowley S. Evaluation of a nurse-led social rehabilitation programme for neurological patients and carers: An action research study. Int J Nurs Stud 2009; 46: 204-219.

6. Janda M, Steginga S, Dunn J, Langbecker D, Walker D, EaKin E. Unmet supportive care needs and interest in services among patients with a brain tumour and their carers. Patient Educ Couns 2008; 71: 251-258.

7. Cifu DX, Carne W, Brown R, Pegg P, Ong J, QutuBUDDIN A et al. Caregiver distress in Parkinsonism J Rehabil Res Dev 2006; 43: 499-508.

8. Cubo E, Martínez Martín P, González M, Frades $\mathrm{B}$, Grp EIEP Impact of motor and non-motor symptoms on the direct costs of Parkinson's disease. Neurología 2009; 24: 15-23.

9. Edwards NE, ScheEtz PS. Predictors of burden for caregivers of patients with Parkinson's disease. J Neurosci Nurs 2002; 34: 184-190.

10. Felming V, Tolson D, Schartau E. Changing perceptions of womanhood: living with Parkinson's Disease. Int J Nurs Stud 2004; 41: 515-524.

11. Gage H, Storey L. Rehabilitation for Parkinson's disease: a systematic review of available evidence. Clin Rehabil 2004; 18 : 463-482.

12. Portillo Vega M.C, Senosiain García J.M $\mathbf{M}^{\mathrm{a}}$, ArantZamendi Solabarrieta M, Zaragoza Salcedo A, NAvarta Sánchez $M^{a}$.V , Díaz de Cerio Ayesa $S$ et al. Proyecto ReNACE. Convivencia de pacientes y familiares con la enfermedad de Parkinson: resultados preliminares de la Fase I. Rev Cient Soc Esp Enferm Neurol 2012; 36: 31-38.

13. Ministerio de Sanidad, Servicios Sociales e Igualdad. Estrategias para el abordaje de la cronicidad en el Sistema Nacional de Salud. Madrid. Ministerio de Sanidad, Servicios Sociales e Igualdad Centro de Publicaciones. 2012.

14. Stanton AL, Revenson TA, Tenneh H. Health Psychology. Psychological adjustment to chronic disease. Annu Rev Psychol 2007; 58: 565-592.

15. Косн T, Jenkin P, Kralik D. Chronic illness selfmanagement: locating the "self" J Adv Nurs 2004; 48: 484-492.

16. KraliK D, Koch T, Rice K, Howard N. Chronic illness self-management: taking action to create order. J Adv Nurs 2004; 13: 259-267.

17. Telford K, KraliK D, Koch T. Acceptance and denial: implications for people adapting to chronic illness: literature review. J Adv Nurs 2006; 55: 457-464.
18. KRALIK D. The quest for ordinariness: transition experienced by midlife women living with chronic illness. J Adv Nurs 2002; 39: 146154.

19. Creswell JM, Plano Clark VL. Designing and conducting mixed methods research. Sage Publications Ltd: London. 2007.

20. Creswell JW. Research design. Qualitative, quantitative, and mixed methods approaches. 2nd edition. Sage Publications Ltd. London. 2003.

21. Giddings LS, Grant BM. Mixed methods research for the novice researcher. Contemporary Nurse 2006; 23: 3-11.

22. Bowling A. Chapter 7: Sample size and sampling for quantitative research. In: Bowling A, Editor. Research methods in health. Investigating health and health services. Edition $3^{\text {rd }}$. Berkshire, UK: Open University Press; 2009: p165-192.

23. Hoenn MM, YAHR MD. Parkinsonism: onset, progression and mortality. Neurology 1967; 17: 427-442.

24. Goetz CG, Poewe W, Rascol O, Sampaio C, StebBINS CT, Counsell C et al. Movement Disorder Society Task Force Report on the Hoehn and Yahr Staging Scale: Status and Recommendations. Mov Disorders 2004; 19: 1020-1028.

25. Chaudhuri KR, Martinez-Martin P, Brown R, Sethis K, Stocchi F, Odin P et al. The Metric Properties of a Novel Non-Motor symptoms scale for Parkinson's disease: Results from an International Pilot Study. Movement Disorders 2007; 22: 1901-1911.

26. Martinez-Martin P, Schapira A, Stocchi F, Sethi K, Odin P, MacPheE G et al. Prevalence of nonmotor symptoms in Parkinson's disease in an International Setting; Study using nonmotor symptoms questionnaire in 545 patients. Movement Disorders 2007; 22: 1623-1629.

27. Miles MB, Huberman AM. Qualitative data analysis: an expanded sourcebook. 2nd Edition. London: Sage publications; 1994.

28. KuPER A, Lingard L, Levinson W. Critically appraising qualitative research. BMJ 2008; 337: 687-689.

29. Whittemore R. Analysis of integration in nursing science and practice. J Nurs Scholarsh 2005; 37: 261-267.

30. Whittemore R, Roy C. Adapting to diabetes mellitus: a theory synthesis. Nurs Sci Q 2002; 15: $311-317$. 